\title{
Dynamic Spatial Positioning: Physical Collaboration around Interactive Table by Children in India
}

\author{
Izdihar Jamil $^{1}$, Kenton O'Hara ${ }^{2}$, Mark Perry ${ }^{3}$, Abhijit Karnik ${ }^{1}$, Mark T. Marshall ${ }^{1}$, \\ Swathi Jha ${ }^{4}$, Sanjay Gupta ${ }^{4}$, and Sriram Subramanian ${ }^{1}$ \\ ${ }^{1}$ Department of Computer Science, University of Bristol, Bristol, UK \\ \{jamil, karnik, sriram, mark\} @ cs.bris.ac.uk \\ ${ }^{2}$ Microsoft Research, Cambridge, UK \\ oharakenton@gmail.com \\ ${ }^{3}$ Brunel University, UK, Mobile Life, Interactive Institute, Sweden \\ mark.perry@brunel.ac.uk \\ ${ }^{4}$ NIIT, Delhi, India \\ Swati.Jha@hiwel.in, Sanjayg@tni.edu.in
}

\begin{abstract}
We present a study of how children demonstrate physicality during collaboration around interactive tables at school. Our results show that children tend to dynamically position themselves around the tabletop area to effect particular social outcomes. These movements around the tabletop allow them to enact coordination strategies in their social interactions with each other to manage their learning and task-based activities. Our analysis indicates the importance of understanding physical strategies and behaviours when designing and deploying interactive tables in classrooms. We discuss how the design of tabletops in school can embrace the extensibility of this technology, providing access for children to shape their own collaboration strategies during learning.
\end{abstract}

Keywords: Interaction techniques, tabletop, spatial formation, dynamic spatial position, collaborative learning, children and India.

\section{Introduction}

Since the introduction of interactive tabletops, we have seen a growing corpus of research exploring how collaborative activities and practices are organised. A key domain of interest in this space is concerned with understanding the collaborative practices of children around tabletops with a view to understand the potential value of these systems within educational contexts. Underpinning this is a body of educational research demonstrating the benefits of peer collaboration in learning activities, and consequently, peer collaboration is becoming an increasingly important concern in the educational experiences of children. What is central to these arguments is that learning is facilitated through encouraging all students to actively participate in exploring learning domains, discussing alternative ideas and perspectives, offering explanations, clarifications and justifications to each other and devising shared goals and plans of action, and thereby facilitating their understanding $[1,13,28]$. In light of these characteristics 
of collaborative learning, interactive tabletops have been argued to have various properties that promote the collaborative nature of these learning activities, for example, the ways that they organise groups around digital learning materials to encourage face to face discussion; how they enable simultaneous participation in collaborative learning, promote engagement and allow all students to share control and responsibility over the input and manipulation of learning materials; and how they make individual interactions at the tabletop visible to support shared awareness [6-8].

Central to these concerns are the ways that the communicative and collaborative practices around interactive tabletops are organised and managed. A significant strand of interactive tabletop research has sought to understand these practices and the factors that mediate them $[5,14,21]$. Such studies, for example have explored the extent to which participation is equally distributed across the group members [5]; the nature of talk in the collaboration [7]; ways in which tasks and activities are spatially partitioned and organised on the surface with a view for coordination and collaboration [5, 23]; the ways that object manipulation and orientation are used in collaborative understanding, communication, task organisation and so on $[12,16]$. These have provided us with a rich understanding and characterisation of tabletop practices.

What is curious has been a tendency to refer to a rather static organisation of collaborators around the tabletop. While this is not an altogether unreasonable state of interaction to examine, we suggest that focusing on situations in which users are immobile underplays features of collaborative activity around tabletops, namely the bodily movement and dynamic positioning of actors around the tabletop for particular social and communicative effect. In highlighting these concerns, our aims are more than simply documenting another interesting feature of tabletop interaction. Rather, we want to highlight how these actions unfold and are produced in situ-with respect to social context of the group, the task and table-and what they achieve in the context of collaborative peer-learning tasks. We also want to explore how such a perspective might inform and refine our ways of thinking about key concepts in tabletop research, such as territoriality, orientation and the spatial organisation of action [14, 24].

To this end, we present a study of children engaged in a peer-learning task around interactive table in a school in Delhi, India. While it is of interest in adding this cultural setting to the predominantly western-based tabletop studies, it is not our intention to focus specifically on cross-cultural concerns. Of greater relevance is that the study is set in a real world classroom and school setting with the accompanying social dynamics of a real collaborative peer learning activity. Within this context, we explore the collaborative action of the peer learning groups and how these actions are produced, understood, and made visible in relation to the spatial and material arrangement of peers, and tabletop artefacts. In particular, we draw attention to a key feature of these practices-namely the dynamic use of spatial positioning around the tabletop. Before moving on to the details of the study, we begin with a look at the related literature to inform our understanding and ground our subsequent discussion.

\section{Related Work}

With the advances of commercially available systems such as the SMART Table and Microsoft PixelSense, the study of multi-touch interactive table use within a learning 
environment has become a growing area of interest. Looking beyond basic usability of such systems, this research has sought to understand the collaborative dynamics of children around digital tabletops and the benefits this might have for collaborative peer learning. One of the key motivating issues here has been on the equity of participation enabled by the multiple interaction access points of interactive surfaces. The shareable nature of these tabletops enables greater engagement by all group members by allowing simultaneous input contribution [20]. Studies have highlighted, for example, how this can have an effect on the nature of conversation during learning tasks leading to greater proportions of task and topic related utterances compared with utterances used for the purposes of task coordination [5, 7].

In Jamil et al. [7], children using interactive tabletops demonstrated task-based, reflective and teamwork related utterances during collaboration. Children went beyond discussing the presented topic and were seen reflecting on a higher level, relating the topic to knowledge and information from other sources (e.g. books and other classes). Such findings have particular significance for the success of collaborative peer learning that is the employment of particular types of talk, such as explanations and clarifications [28]. Similarly, the SIDES application [21] was able to motivate and support effective group skills between children with Asperger's Syndrome (who find working in groups difficult) in a four player cooperative game. Their findings include an increase in positive language use and a decrease in aggressive behaviour, building confidence in teamwork and offering a sense of participation and belonging.

Multi-touch systems themselves offer a different kind of platform for collaborative interaction compared to single touch systems. They allow users to spatially position themselves and simultaneously manipulate objects. In a single touch condition, only one person can manipulate an object at any time. This may not be a conducive environment for spatial movements to occur naturally: Harris et al. [10] suggest that single touch tends to result in a more turn-taking conversation that may indicate a turntaking behaviour to follow these conversation patterns. Furthermore several researchers have shown that bodily movements are not seen when groups of students perform classification and sorting tasks around a non-digital tabletop [13,25]. One possible explanation could be that the experience of interacting with digital and physical objects differs: Marshall et al. [25] report that children in digital tabletop conditions were "more forceful" compared to the children in the non-digital conditions when blocking access to objects. This suggests that children may adopt different strategies when presented with different tabletop settings.

Other studies have focused on interactions on the tabletop and the embodied gestures around tabletop objects in collaborative activity. Visibility of these interactions in the face to face interaction of tabletop computing for example has been argued to promote an awareness of actions to others $[6,15]$. This can be important in task coordination, but is also important in other features of peer learning such as consensus building, delegation of action, and production of explanations. Nacenta's work [23] shows how awareness in tabletop communication and collaboration is supported by three key mechanisms: territoriality, the division of the table surface into personal and shared regions for interaction (cf. [14]); feedthrough, namely the public visible manipulation of artefacts on the tabletop, for example Kruger et al's [14] notion of 
object orientation in communication; and consequential communications [25], the embodied actions produced by the hands, arms and bodies of group members as they collaborate. Let us consider these further.

The most significant work of territoriality in tabletop interaction can be seen in the seminal work of Scott et al. [24], in which the tabletop area is divided into four spaces: personal, shared, group and storage. The personal territory is that closest to a person and can be viewed as an extension of oneself [14, 24]. A participant can disengage with his or her group while exploring ideas before sharing it with the other members. Shared space occurs when a sub-group is formed and uses a particular area on the tabletop to direct their attention and discussion. The group space, usually located in the middle of the tabletop, is a communal area where all the members can jointly explore concepts together. The storage space serves as a holding area of unused resources and can be located anywhere around the tabletop. This partitioning influences how group members coordinate their activities when dealing with objects. Importantly, Scott et al. [35] make distinctions of territorialities along functional and spatial dimensions. In this sense, the exact size and position of these territories are socially constructed within the context of the task and setting. However, they also characterise these territories in terms of their relationship to the seating positions of the group. Implicit in their characterisation and representation of these spaces is a static set of seating arrangements. This presents strong implications for tabletop design to orient to territoriality in terms of its spatial rather than functional characterisation. While this is not an entirely unreasonable assumption, we want to argue that it at times ignores the potentially important role of dynamic bodily positioning around the tabletop in the construction of territorial arrangements of learning tasks. It is interesting to question how territoriality might be characterised when it is less bound and determined by fixed seating positions [14, 24, 27].

Within the context of these territorial practices are the feedthrough issues of orientation management-the degree to which objects on the tabletop are oriented to particular individuals or groups of collaborators: the orientation of an object on a tabletop towards an individual can confer certain territorial responsibilities that allow users to partition responsibility for particular features of the task. Such arrangements are bound to particular social protocols regarding who can manipulate particular objects on the tabletop within different territories. In this respect, interacting with objects by repositioning or re-orienting them is a negotiated concern among the collaborators. Within this context, orientation is actively used as a resource to achieve particular social effect and meaning (Kruger et al. [14]).

For Kruger et al., orientation of objects on tabletops serves three critical roles during collaboration: comprehension of information, coordination of actions and communication between members. Thus reorienting an item is actively used for a variety of social and cognitive concerns, for example, to invite comment, hand over responsibility, initiate action, clarify intent, enable shared reading among collaborators, establish conceptual relationships among items, and so forth. What emerges from this is the situated production of meaning through orientation, that is meanings can be enormously varied. With such varied inscription of meaning through orientation, there are also potential points of conflict, in particular for exploratory and expressive learning 
tasks where spatial organisation of artefacts is also bound with conceptual understanding of the learning domain. Although useful, Kruger et al's explanation perhaps underplays the other means through which we may construct our relationship with objects on the tabletop and other members. Rather than creating meaning by orienting objects on the table, people can also simply reposition themselves with respect to the objects and other people: a feature of tabletop interaction that has been underplayed.

The work of Tang [27] provides us with more insight into spatial positioning. This highlights a number of spatial positions adopted by collaborating couples. These positions are used to achieve greater or lesser closeness of collaboration and avoid territorial conflict with couples adopting positions of closer proximity when working in a tightly coupled way. While Tang's work is broadly in line with the concerns of our paper, it presents a relatively abstracted characterisation. In this respect, we miss out on how the detailed use of bodily positioning and orientation at the tabletop operates as an embodied and situated practice, to be combined with other gestures and actions in relation to tabletop artefacts and collaborators, and the particular social meanings these practices achieve. The work of Yamashita et al. [29] provides us with a richer account of positioning and bodily orientation along these lines. This work is concerned in particular with how such action is organised around tabletops in distributed collaborative settings and how different video configurations and bodily views better enable the achievement of mutual orientation of awareness. As such it is less concerned with how such practices are achieved in collocated settings and in relation to the specifics of collaborative learning tasks. In the context of computer supported collaborative learning such accounts can be found in the work of Suzuki et al. [26]. Suzuki highlights the importance of seeing learning as participation in a culture of practice and the importance of designing to facilitate participation and interaction among learning groups. This is achieved through the use of gaze, body movement and gesture in relation to the computer artefacts. The study examines the interactional details of these practices in relation to group learning around a tangible computer interface. In this paper, we extend these concerns to consider how such interactional practices are organised in tabletop learning.

In informing our concerns we can also draw on some more general accounts and theories which while not specific to tabletops can nevertheless help frame our interpretations. Of particular significance here is Goodwin's [3] treatment of pointing as a situated and collaborative practice. For Goodwin, "pointing is constituted as a meaningful act through the mutual contextualization of a range of semiotic resources including at least, 1) a body visibly performing an act of pointing; 2) talk which both elaborates and is elaborated by the act of pointing; 3 ) the properties of the space that is the target of the point; 4) the orientation of relevant participants toward both each other and the space that is the locus of the point; and 5) the larger activity within which the act of pointing is embedded." Of significance is the collaborative nature of pointing and the multiple spaces and meaning making structures (for example artefacts, bodies of the actors) that are brought together to achieve it. Furthermore, this work starts to give us insights into some of the different purposes of these pointing (for example explaining vs directing action) and how is organised to achieve these effects. Kendon's work on gesture and spatial positioning [10] on the f-formation is 
also relevant. In this, when people communicate with each other, they mutually form f-formations (facing formations) that create mutually accessible transaction segments (o-spaces) between each participant. Attention and interaction are typically focused in this space with ongoing spatial and postural work being used to try and maintain these spaces. Indeed some other recent work in tabletop interaction has looked at spatial positioning based on Kendon's F-formation [10], most notably in Marshall et al. [17]. In contrast to the work we present in this paper, the concerns of the Marshall study were more with gross observable outcomes to see where social interactions did or did not work, rather than the detailed and ongoing production of social action. Our study builds on the concerns outlined here and explores the embodied and situated nature of collaborative learning practices around an interactive tabletop. In particular, it seeks to highlight the role and meaning of bodily positioning and orientation for the specific demands of collaborative learning, and this is reflected in the study that follows.

\section{Study Description and Analysis}

Participants. We recruited 51 pupils (between 11-13 years old) consisting of 27 females and 25 males from a local school in Delhi, India. The participants were divided into 11 groups of 4 to 6 pupils, a typical number for group-based classroom activities in these schools and fulfils the criteria for working in small groups [2]. This school is a private fee-paying school with mixed-sex students from different backgrounds. School children had access to a computer on regular basis. The medium of education is English and students talked in English during the entire study although they were not asked to speak in a specific language. The assignment of groups was conducted in consultation with the teachers in order to create groups of compatible ability levels.

Apparatus. We produced two custom-built FTIR tables [4]: $90 \mathrm{~cm} \times 90 \mathrm{~cm}$ and $76 \mathrm{~cm}$ high with a projection of $72 \mathrm{~cm}$ x $48 \mathrm{~cm}$ (NEC projector). Point Grey Dragonfly 2 infrared cameras tracked user interactions. The task applications were created using Adobe Flash and Action Script 3. Tables were configured with two interaction techniques: (1) direct touch: a multi-touch platform where multiple users could interact directly with digital objects; (2) pantograph: finger movements in the 4 pantograph areas are amplified to create larger cursor movements on the surface, allowing digital objects to be reached without stretching across the table [18]. For larger groups, additional pantograph areas were added. Both tables were situated in the computer room at the school for the three days of the study. Chairs were available around the table.

Data Collection and Analysis. During sessions, children were exposed to both interaction techniques and learning activities, and after each session we switched the order of the conditions randomly. Video was used to record the physical and verbal behaviour of all the groups. Our analysis draws on a detailed examination of the video recordings and focuses on the collaborative achievement of the tasks. We describe the interaction details of how gestures, talk and action are produced, coordinated, made visible and understood with respect to the table and on screen objects. We used evidence-based interpretation to illustrate our findings and observations following the 
methodology of existing literature on interaction analysis [9]. The empirical data include sequence of vignettes depicting the children's interaction with digital objects.

Tasks and Techniques. Two collaborative learning activities were deployed: (1) spider diagram: similar to a mind map in which a topic is investigated and explored by visualizing associations and relationships between key concepts (Fig. 1a), and (2) classification: to classify and group twenty elements (images and concepts) of a topic according to category (Fig. 1b). Each category is represented by a square yellow box. The layout of the elements were scattered in a circle around the centre point of the surface providing equal access to the elements for each participant. The spider diagram and classification activities were based on "Photosynthesis" and "Animals", both topics in the Indian National Curriculum (further description in [7-8]): using different learning activities and interaction techniques were intended to add richness to the study, and ensure that these findings were not dependent on a particular educational topic or interaction method.

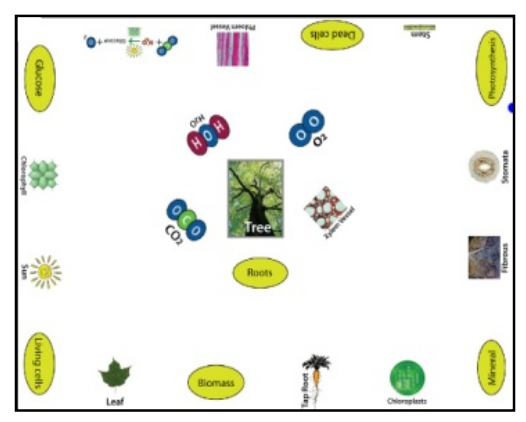

Fig. 1a. (L). Spider diagram (direct touch technique)

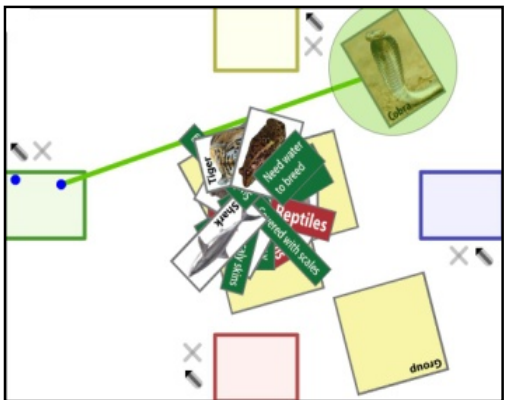

Fig. 1b. (R). Classification (pantograph technique; blue dots represent the user's fingers)

A number of features about the tasks and technology are worth raising here as they have particular implications for the interpretation of the spatially organised practices of interaction observed. First, the task at the outset is essentially orientation-free, i.e. the arrangement of items on the tabletop neither suggests nor commits the learning group to any particular spatial organisation. Second, the task itself has a spatial component to it- spatial relationships between tabletop items can and are used to explore conceptual relationships between items. While spatial structures are not imposed, spatial parameters such as position, mutual proximity and orientation can be used to achieve mutually understood conceptual organisation of the information. This is a standard feature of educational tasks that involve things such as classification or conceptual organisation of information. In the direct touch system, no interface elements favoured a particular spatial organisation of the group around the table. In the pantograph system, the elements at each side of the table could potentially encourage a particular spatial positioning of the students to act in controlling the system. With chairs positioned at the table the students were free to sit or stand and had room to 
move around. The dimensions of the table generally allowed the children to reach all parts of the table though for some this would require stretching to reach the furthest part. Notably there was nothing in the task, interface or hardware set up that was purposefully designed to induce the learners to move around the tabletop.

\section{$4 \quad$ Results}

In this first vignette, the group is discussing some initial classification for the structuring of roots, stem and leaf. P1 said: "Put the roots next to the tree right." At this moment, P1 gestures above the table to trace a path from where the "roots" piece is located to next to the "tree" piece (Fig. 2a).

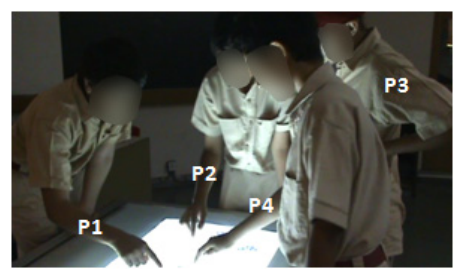

Fig. 2a (L). P1 pointing at roots above the tabletop

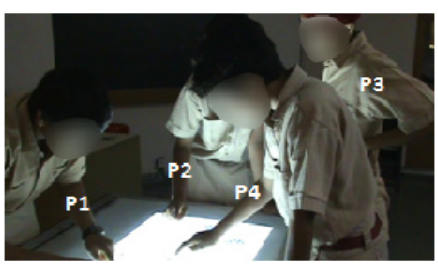

Fig. 2b (R). P1 moves toward the corner

In doing this, P1 is suggesting a conceptual organisation on this part of the task, these pieces being within easy access of his current position. While he is doing this, P2 and P4 are both attempting to impose their own classification structures on the same pieces in the middle of the table, blocking each other's finger gestures as they compete to organise the pieces. P2 lets P4 complete his positioning, but then lifts P4's hand off the table to prevent any further interaction, allowing P2 to then bring another piece over to the emerging structure. P1 responds and says "Don't you think we should put leaf there so we can put roots over here?" [pointing to the appropriate locations on the tabletop to suggest a particular spatial and conceptual organisation]. P2 then suggests "Put roots here." (but moves the "roots" piece to a different location). Without saying anything, P1 moves further across the side of the table to position himself more in line with the newly positioned piece (Fig. 2b). In doing this P2 has built on P1's suggestion but through his action suggests a slight modification to the spatial structuring that they should use

A number of key things can be seen here. First, through this action and the continuation of work there is a non-verbalised demonstration of consensus with the structure offered by P2. Second, the continuation or work (and thereby the consensus demonstration) demands a particular bodily orientation with respect to the information artefacts on the table (Fig. 2b). We see a two handed manipulation as objects are brought into conceptual relationships with each other. Such a 2-handed manipulation would be awkward without moving either the artefacts or the body. Moving the artefacts was not a viable option since their spatial positioning was given meaning in the 
context of the task. To move them would have disrupted the emerging spatial structure, undoing progress and importantly, socially going against the proposed structure.

As P2 makes this manipulation, P2, P3 and P4 are focused on the other part of the table as they discuss other parts of the task. While there is distribution of the task activities here, they are not entirely separate and indeed there remain dependencies on the work being carried out by P1. In response to this, P2 breaks away from P3 and P4 and moves towards the corner of the table and reorients his body to be in line with P1 and the artefacts that P1 has been working on and asks "Pl where did the leaf go?". As P2 asks the question he inspects the area where P1 has been working in order find the leaf and see what P1 has been doing. Both positioning and orientation are key at this point both for the cognitive and social demands of the task. Firstly P2 does not position himself right next to $\mathrm{P} 1$ but rather remains in a position somewhere between P1 and the other two participants-his engagement is temporary and maintains links across the subgroups. While in this corner position, his bodily alignment with the tabletop artefacts make the necessary comprehension and search easier. This is consistent with Kruger's articulation of orientation but as we again see in this instance, rotation of the tabletop structures is not possible as these are bound up with the spatial representation of concepts central to the learning activity at hand. As such orientation of the body is used instead. An additional feature of this orientation is to initiate a discussion with P1. Indeed what follows is that P2 leans in to move one of P1's pieces which then bring the work in line with the other three had been working on.

\subsection{Orchestrating Participation}

With the movement of P2 to the corner, we see an interesting behaviour arise from P3 and again this is one that is a key feature of such collaborative learning tasks. In moving to the corner, $\mathrm{P} 2$ actually creates some open space. Up until this point P1, P2 and $\mathrm{P} 4$ had been adopting positions along the sides of the table and as such were more actively involved both in the task both in terms of manipulating tabletop content and in terms of conversational contribution. P3 had been positioned slightly outside the primary f-formation made up by the other three participants and as such was struggling to get involved. With P2 moving and opening up a space between P2 and P3 (see the space created from Fig. 2b and 3a), P3 then moves closer into the table and spreads his arms across both sides of the table to establish and protect his position at the table, albeit a corner position (Fig. 3a). From this point, P3 is able to actively contribute to learning task through manipulation and conversation (Fig. 3b). Such behaviour is worthy of some comment here because it highlights the importance of being involved in the learning task and some potential difficulties of orchestrating such involvement with tabletop computing. Being actively involved in the task can be more engaging rather than passively watching. From a learning perspective, it allows the participant to explore their own conceptual contribution and have them questioned and critiqued by peers. But there are potential social concerns at play also in relation to participation in such learning tasks. For example, it can be important to demonstrate your attempts to contribute and demonstrate your intellectual grasp both to peers and potential assessors. While it is difficult to comment on the exact motivation 
in this particular context there are a number of things which are clear from the behaviour. First of all was how participation was inhibited by the particular form and size of the table that at times made it physically and socially difficult for some to manoeuvre into a position of participation. Second, the opportunistic spatial orchestration of participation by $\mathrm{P} 3$ demonstrates its importance to him in this learning context and for any number of the learning related reasons outlined above. As this is happening P4 carries out some manipulations in the middle of the table, then calls out, $\mathrm{P} 4$ said: "The sun goes to the tree!" [as he speaks he touches Sun and then touches Tree. P2 removes his hand from the middle of the tabletop. P4 then draws a line between those two keywords. P3 looks in the direction of the tabletop].

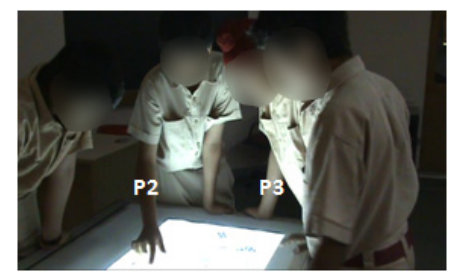

Fig. 3a (L). P3 moving closer to the tabletop

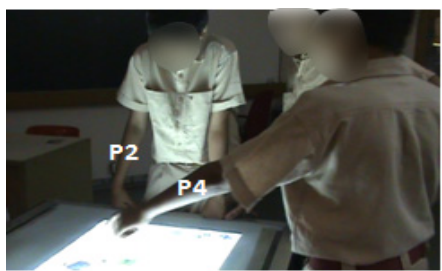

Fig. 3b (R). P4 suggesting a new location

P4 said: "And now branch is here... and then leaf and roots here!" [P4 moves his right palm above the tabletop towards the corner right side of the tabletop, and the moved it again towards the corner of the left side of the table top and then towards the bottom of the tabletop. All the children then start to move objects from the middle of the tabletop towards the side of the tabletop leaving Tree in the middle. They were also scaling and rotating objects for visibility and orientation within this representational structure.] What is happening here is that a conceptual structure for the task is gradually coming into place as represented through an agreed upon spatial organisation. While the structure is not complete, by agreeing to the conceptual organisation it allows them to plan for how the task can be appropriately divided up. The movement of the objects to particular spatial locations is towards this end. It is only having got to this point through their spatial explorations that they can divide the task up in this way. Their arrangement of the objects in particular locations on the tabletop corresponds to this distribution of labour. Given this arrangement, what is curious at this point is how the enacted task distribution does not simply map onto their existing spatial positions. Rather they begin a sequence of delegation and volunteering to take on the subtasks that have been created.

This is initiated by P4 who says "Okay, now, now I will do the roots stuff!". As we see in Fig 3b, P4 gestures towards an area of the table as he speaks and as he is doing this he is turning his body away to begin to move towards the area where the roots are (next to P2). As he is moving away he begins to delegate with an utterance and a gesture. He does not need to complete the delegation as the remaining boys jump into to try and get first digs on the other parts of the task. Both P2 and P3 simultaneously say "I'll do the stem", P1 following with "leaf". As these utterances are taking place P4 continues to move round towards P2's old position, while P2 and P3 both begin to 
move round the table to make way for P1 and also manoeuvre themselves into the correct position at the table to deal with the "stem" work (Fig. 4a). Of particular note is that P2 and P3 continue to move around until P2 is in prime position for "stem", that is, $\mathrm{P} 3$ does not stand his ground but moves further along the table and gives into $\mathrm{P} 2$. Again, P3 is moved out of the way to a position where it is difficult for him to participate. Realising that he is spatially no longer in a position to actively participate. P2 moves round to stand between P1 and P4. From here he is able to actively get involved (Fig. 4b).

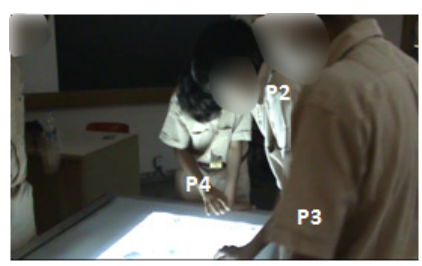

Fig. 4a (L). P2,P3 and P4 spatial positioning

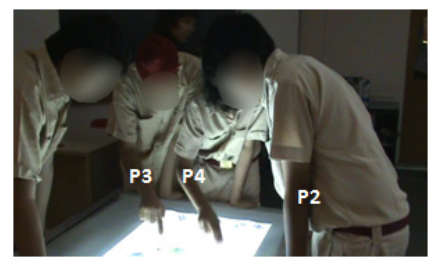

Fig. 4b (R). P3 shifting

What we can say about this intriguing sequence of actions is that the distribution of learning tasks is not one of simple spatial convenience whereby the learners are taking on the tasks that are closest to them. And indeed, the spatial representation of the conceptual relationships is manifest in order to be meaningful to the learning task as opposed to be organised around spatial convenience for interaction. Furthermore, these set of subtasks are evidently not considered equal and while it is difficult to ascertain the basis of such differentiation, it is clear from the behaviour that the participants consider it to be the case. This could be a question of certain parts of the task being more interesting to particular individuals, more suited to specialist knowledge of individuals, more intellectually demanding and therefore suited to certain individuals. Whatever these particular values, we can see too that certain social relations are being enacted through these spatial positioning and the ways in which peers displace or give way to the other peer collaborators. Again, we can only speculate as to the details of these but this does highlight is how such learning tasks in the real world do not exist in some social vacuum but within a whole range of existing social and intellectual relations between the peer learners that are an inherent part of these practices in real educational settings.

\subsection{Orientation, Explanation and Clarification}

Here, a different group is exploring the process of photosynthesis (initial positionFig. 5a). P5 said: "Look. This is the entire process of photosynthesis!" [P5 points to a group of keywords $\left(\mathrm{O}_{2}, \mathrm{CO}_{2}\right.$, Phloem, Photosynthesis) in the bottom lower right hand corner of the table (relative to him) using both of his index fingers (Fig. 5b)].

P5 said: "All of this goes into photosynthesis!". He then traces an arc with both of his hands and turns his body at the same time to a second group of keywords 
(Mineral, Sun and a few other non-visible keywords) at the bottom left corner of the table (relative to him). This arc and bodily movement are performed while looking at P3 and P4 with P1 and P2 following his movement. P5 then repeats the movement and said: "This is all the same. This is photosynthesis!". P1 then answered: "Oh my God! You're smart!". P5 then added: "maybe bring all this here" [he traces the same arc in reverse direction from his lower left corner to the lower right corner of the table]. P5 then continued: "We need to move everything there!"
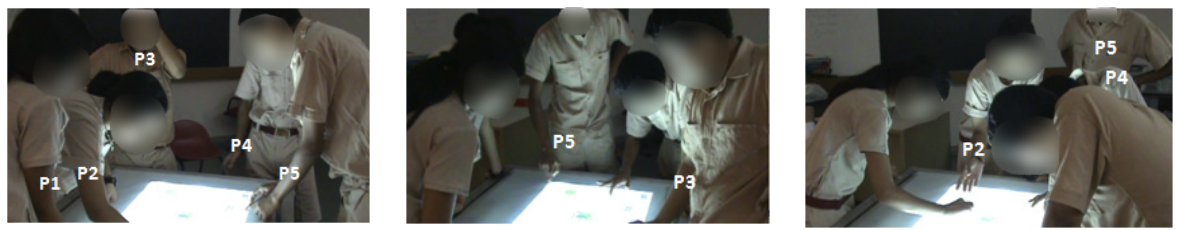

Fig. 5a (L). Initial position. Fig. 5b (M). P3 and P5 moving Fig. 5c (R). P2 shifting position

In this set of interactions we see that P5's conceptual breakthrough establishes a certain spatial order on the tabletop representations. The others, being impressed with this conceptual insight, then commit to further establishing the spatial relations this entails and begin to move the pieces to the location P5 has suggested and orient them in a way that is commensurate with the established grouping in the lower right hand corner of the table (relative to P5). This then commits the group to a new orientation. P3 who has moved to access the keyboard to the left of P5 then says to P5 "Move that side" and gently nudges him out of position, which continues the instruction. P5 initially resists but then moves round to P3's original position (Fig. 5b). This new position for P5 is also at the correct orientation for the spatial representation of the conceptual organisation suggested by P5. This allows him to continue the intellectual lead that he has set. P2 and P4 who are either side of P5 continue to work on the arrangement under the direction of P5 who is watching them. P5 stands back from the table with hands on hips, visibly indicating that he is letting the others work on the arrangement. At this point $\mathrm{P} 4$ goes to place the $\mathrm{CO}_{2}$ incorrectly. P5 then takes action to intervene. He moves in closer to $\mathrm{P} 4$ and aligns himself with $\mathrm{P} 4$ just partially behind P4. As P5 moves he points to a location on the tabletop he says " $\mathrm{CO}_{2}$ is here, here!" (Fig. 5b). What we see here is a key use of bodily positioning and orientation in collaborative learning for the purposes of explanation and direction. P5, as the person doing the explaining aligns himself both in relation to $\mathrm{P} 4$ (to whom he is explaining) and in relation to the spatial arrangement on the tabletop being referenced (Fig. 5b). Immediately after this sequence we see a related practice that is again of significance in relation to collaborative learning, namely questioning conceptual organisation and inviting clarification. Here P2 moves from her original side of the table and positions herself in front of P5 and P4 and facing the spatial representation being worked upon (Fig. 5c). P5 stands back to allow her into position.

P2 said: " $\mathrm{O}_{2}$ is .... Wouldn't $\mathrm{O}_{2}$ be after photosynthesis?" [she makes a gesture to indicate this suggested temporal relationship between $\mathrm{O}_{2}$ and photosynthesis]. P5 then said: "No." Here then, P2, who is seeking an answer to a conceptual question, 
actively uses the alignment of herself with the spatial representation of concepts on the tabletop and with the person from which she is seeking clarification, P5.

\subsection{Pantograph}

We also noticed similar behaviour when the students used the pantograph technique. This is very peculiar as the main benefit of using the pantograph is the ability to reach far object without the need to move. Physical movement and positoning from one place to another is hardly necessary and yet the children demonstrated such. In the interaction below, we see two children from Group 4 move from their location to help another child resize a Group Box. The Group Box is an essential part of using the keywords as it provides a space where the children can classify and group the animals, collectively signalling their understanding of the task.

P2 said: "You just made it bigger!" [referring to the Group Box]. Immediately after he does this, P1 hovers his left hand just above P4's pantograph (where P4's hand was located). P1 then moved closer towards P4's location and at the same time moved his right index finger from his pantograph towards P4's pantograph. P4 then released his touch on his pantograph. P1 touched P4's pantograph, highlighted the Group Box and started to scale it down. However, after some 20 seconds or of P1 scaling the Group Box without much success, the Group Box still had its original size.

P2 said: “Make it smaller! Make it smaller!”. At this point, P3 stood up and moved towards P4's pantograph area. P3 stretched his left hand and touched his index finger on P4's pantograph area, which he used to scale down the size of the object. P2 then said: “He's a genius!". P1 then moved closer to P4's position, touched P4's pantograph and further scaled down the object. What is apparent from the interaction above is that P1 and P3 both moved position and used P4's pantograph to: 1) help and assist P4 and P1 to perform an action (in this case, scaling down a Group Box), and 2) to stop P4 from doing something that is visibly seen to go against the group's decision (i.e. making the Group Box bigger). What is curious in this interaction is that neither P1 or P3 used their own pantograph in order to resize the Group Box despite the available facilities to do so, but move and position themselves near P4's pantograph area and subsequently use this to perform the action. Given that this was previously his personal working area, it is notable that $\mathrm{P} 4$ showed little resistance to this.

We speculate a number of possible reasons for this behaviour: 1) the children perceive territoriality and ownership of an object based on the object's location. In this case, the Group Box is located within P4's personal space, hence P1's and P3's movement towards that area, 2) as territoriality of Group Box was mapped to belong to P4, perhaps using P1's and P3's pantograph would considered to be rude and an act of intrusion of object ownership, 3) the orientation of objects may determine the physical positioning of a user, in this case the position of Group Box was orientated towards $\mathrm{P} 4$, and 4) the children were demonstrating meaningful social attributes (for example teamwork), in particular, demonstrating the case of being supportive and helpful with each other, even though there was no verbal request for help from P4 to concur with the decision to resize the object. The ability of the pantograph to address physical 'reach' of digital objects outside of a user's arm length would seem to make 
such movements unnecessary, and given their experience with the pantograph by this stage in the study (and the fact that they use P4s pantograph), it cannot simply be that they are struggling with the interface. Clearly, spatial physical movement carries value above and beyond the physical necessities of interface.

\section{Discussion}

Through the fieldwork, we have begun to highlight the situated and embodied practices enacted by groups of students from Delhi performing collaborative peer learning activities on an interactive tabletop. In particular, we have focused on the ways that these learners employ dynamic bodily orientation and positioning around the tabletop for particular social, cognitive and collaborative effect in the context of these collaborative learning tasks. Such actions are made meaningful in the particular context of learning and are collaboratively organised in relation to the other learners, the tabletop and the task based artefacts on the tabletop. While such bodily arrangements and positioning are a resource in any collaborative activity, what is of interest is the particular ways that they are manifest in the context of these tabletop learning activities, why they are important in these activities, and the particular implications this has for our theories and design of tabletop interaction.

Central to the arguments we wish to make here is the cognitive use of spatial representations that is fundamental to such exploratory learning activities and tasks [11]. Within these tasks, domain knowledge comes to be explored and represented through the spatial relationships between the tabletop entities. Orientation, position and relational proximity between these different entities are all parameters through which the learners collaboratively come to represent key conceptual relationships in the knowledge domain both as tentative propositions to discuss and ones to which the learners eventually commit to as more static representational structures. Significant here is that such spatial representations of knowledge are emergent features of the learning task as it proceeds towards some form of resolution. Such an additional layer of spatial meaning then is another concern- embodied actions of the learner must be organised and that takes us beyond the key theoretical apparatus of existing tabletop research. For example, while territoriality remains an important and demonstrated concern within tabletop collaboration that has its expression in spatial relationships among actors and artefacts, this expression is made considerably more nuanced as actors orient to the additional layers of spatial concern in these tasks.

Similarly, as we have seen in the findings, the spatial configurations of actors around the tabletop requires a richer account than simply the management of relative closeness or looseness of collaborative coupling, though this is indeed important. With regards to Kruger et al. [14] articulation of object orientation in tabletop collaboration, our findings would appear to align with a number of the theoretical concerns of this work. That is in the role of tabletop object orientation in comprehension, communication and coordination work. But as we have seen in these learning tasks, the orientation and positioning of objects takes on additional significance in the form of the spatial representation of knowledge. In this respect the orientation of objects is 
but one resource that can be used to achieve different forms of comprehension, communication and collaboration work but is one that is not always available as objects gradually adopt their positions in the spatial representation of knowledge. As such, bodily positioning and orientation with respect to the objects becomes an additional resource through which this kind of work is achieved. Such a resource can be especially important in the management of orientation and positioning in the real world learning contexts here where the larger group sizes configured around the tabletop present more complex orientational and positional dependencies between the actors and artefacts which need to be managed. With this in mind, then, let us consider some of the more specific ways in findings that this use of bodily orientation and positioning around the tabletop was used and how these have particular significance in the context of the collaborative learning activities:

1) In the data, a very pragmatic concern arises from the need to manipulate multiple objects into meaningful spatial relations with each other to represent conceptual relationships. When conducted as a bimanual activity, this entails certain physical demands on bodily orientation and positioning in order to bring objects together.

2) Bodily orientation and positioning was used to facilitate the interpretation of conceptual relationships being expressed spatially by the other learners. This involved alignment with the orientation of these particular spatial structures, but to do so in ways that also maintained orientation to other structural features of the representation. This allowed interpretation of sub-parts of the task in the context of the whole.

3) Movement and positioning was used to initiate enquiry into particular conceptual representations, one of the significant features and benefits of collaborative learning. In doing this, bodily orientation and positioning is organised both in relation to the information and the other learners involved. This also sets up the possibilities and permissions for subsequent manipulation of the spatial representations created by others allowing them to build and refine the conceptual work being performed.

4) Positioning and orientation are used to orchestrate and enable participation. Of significance here is that with the larger number of learners in these real world learning groups, some learners end up in positions around the table where they are disadvantaged in their access to the active transactional spaces in play. In such circumstances, they opportunistically seek positional openings as they arise in the positional configurations of the group. Such participation is key to learning in these groups allowing individuals to explore their own conceptual understandings. Likewise, such visible attempts to participate are not only important in demonstrating contribution to the group but in the context of real world educational settings may be important to assessment. Furthermore, such opportunities to participate and contribute are emergent in the context of the task itself. Only as the task progresses do it become apparent and mutually agreed how the task can be divided up into subtasks. Here again, positional manoeuvring can be used to orchestrate participation and ensure influence over the emerging transactional spaces. In such manoeuvring, it is not simply the characteristics of the task and artefacts that are brought to bear on action, but also the preexisting social relations of the group members from the broader school experience itself that brought forth a whole host of existing social relations among the learners. 
5) While positioning and orientation enables participation, this goes beyond the interaction technique settings, i.e. the children violate the "supposed" working pattern when using the pantograph technique. Thus, although the pantograph allowed equitable access to objects within the tabletop area [18], students can be seen using other people's pantographs. Of significance here is the value that the orientation and positioning brings forth during the activity. Physical movement allows the children to continue with the activity without interrupting the existing spatial flow and relationship that is crucial in their learning. Perhaps positioning themselves at another member's pantograph also ties in with territoriality and orientation notions above. In one hand, the children wanted to help another member as part of demonstrating group cohesiveness, while on the other hand they were aware that the object does not belong to them (i.e. it was not orientated towards them and not located within their personal space $[14,22]$. They respected the notion of ownership and thus moved themselves accordingly in order to gain ownership in the process of helping others.

6) Finally, we see the important use of physical orientation and repositioning in relation to key features of collaborative learning, namely to direct and explain, and to invite clarification of proposed conceptual structuring. Notable here was that not all participants have equal status within the groups at different points in the task. Rather at any time, certain individuals may have key insights that emerge that lead to particular spatial representations and around which the group spatially converge and achieve consensus. This can demand the intellectual lead be positioned at the dominant positioning in the transactional space. Key here is that in directing and explaining the concepts to others, the person directing or explaining will align themselves with both the spatial representation and the individual explainees so as to shape the explanatory gestures and talk to the perspective of the explainee. In a similar vein, we see those inviting explanation and clarification adopt similar mutual alignment with the representation and explainer to allow both articulation of their misconceptions and appropriate receipt of explanation.

In evidence here then is the situated and collaborative achievement of these significant features of collaborative learning practices. In such practices, and to paraphrase Goodwin [3], bodily orientation and positioning around the tabletop are constituted as meaningful acts through the mutual contextualization of the semiotic resources to hand and involves the orientation of participants to each other and the representational space of artefacts. What we want to argue here, is that such actions are an important part of a repertoire of resources available for such meaning-making much in the same way that, for example, Kruger et al's [14] orientation of tabletop artefacts might be regarded. In many aspects of these spatial and exploratory learning tasks they offer some potentially significant benefits when other resources (such as object manipulation) may be constrained or unavailable.

\section{Design Implications and Conclusion}

As well as the theoretical implications we have discussed above, there are particular implications for how we approach the design of tabletop interactions for these settings in ways that enable rather than constrain these important actions. For example, some applications and interaction mechanisms impose certain location dependencies on the 
users that may make them less flexible in their use of spatial positioning and orientation around the tabletop. For example, the idea of user identification and object ownership at one designated location (i.e. A stays at location $\mathrm{X}$ throughout the task with her personalized tools) or the capability of restricting user input (such as the features available in [21]) may impose restrictions along these lines. Using a fixed and digital control bar as suggested by Olson et al. [19] may impose constraints for similar reasons. What may be important is for applications to be fluid, dynamic and moveable. Mobile storage bins [29] and adaptive personal territories [12] may provide some interesting solutions here where learners have the capability of grouping objects (in our case keywords and pictures) and moving them around as they position themselves around the tabletop. But there remain questions over such whether storage bin/personal territories move automatically as the children move or whether they should be moved manually by their owner. In raising these our intention here is not to be overly prescriptive in the design suggestions nor to suggest that location-bound interaction mechanisms on the tabletop entirely prevent the use of bodily orientation and positioning as a resource (indeed, even in the positional constraints imposed by the Pantograph we see some deployment of these activities). Rather, our intention is to highlight the importance of these action resources in collaborative learning around tabletops and prompt reflection on how particular interaction mechanisms may constrain or hinder their enactment.

Acknowledgements. We would like to thank KPT (810502055410) and UPM (T01839) in Malaysia for partially sponsoring this research. A huge appreciation also goes to the Bristol Interaction and Graphics Group, NIIT, all of the teachers, staff and participants.

\section{References}

1. Eisenberg, A.R., Garvey, C.: Children's use of verbal strategies in resolving conflicts. Discourse Processes 4, 149-170 (1981)

2. Engleberg, I., Wynn, D.: Working in Groups. Communication Principles and Strategies. Houghton Mifflin Company (2000)

3. Goodwin, C.: Pointing as Situated Practise. In: Kita, S. (ed.) Pointing: Where Language, Culture and Cognition Meet, pp. 217-241. Lawrence Erlbaum (2003)

4. Han, J.Y.: Low-cost multi-touch sensing through frustrated total internal reflection. In: Proc. of UIST, pp. 115-118. ACM (2005)

5. Harris, A., Rick, J., Bonnett, V., Yuill, N., Fleck, R., Marshall, P., Rogers, Y.: Around the table: are multiple-touch surfaces better than single-touch for children's collaborative interactions? In: Proc. of CSCL, pp. 335-344 (2009)

6. Hornecker, E., Marshall, P., Dalton, N.S., Rogers, Y.: Collaboration and interference: Awareness with Mice or Touch Input. In: Proc. of CSCW, pp. 167-176. ACM (2008)

7. Jamil, I., O'Hara, K., Perry, M., Karnik, A., Subramanian, S.: The Effects of Interaction Techniques on Talk Patterns in Collaborative Peer Learning around Interactive Tables. In: Proc. of CHI, pp. 3043-3052. ACM (2011)

8. Jamil, I., Perry, M., O’Hara, K., Karnik, A., Marshall, M.T., Jha, S., Gupta, S., Subramanian, S.: Group Interaction on Interactive Multi-touch Tables by Children in India. In: Proc. of IDC, pp. 224-227. ACM (2010) 
9. Jordan, B., Henderson, A.: Interaction Analysis: Foundations and Practice. The Journal of Learning Sciences 4, 39-103 (1995)

10. Kendon, A.: Conducting interaction. Patterns of behavior in focused encounters. Cambridge University Press (1990)

11. Kirschner, F., Paas, F., Kirschner, P.A.: A Cognitive Load Approach to Collaborative Learning: United Brains for Complex Tasks. Ed. Psy. 21, 31-42 (2009)

12. Klinkhammer, D., Nitsche, M., Specht, M., Reiterer, H.: Adaptive personal territories for co-located tabletop interaction in a museum setting. In: Proc. of ITS, pp. 107-110. ACM (2011)

13. Kruger, A.C., Tomasello, M.: Transactive discussions with peers and adults. Developmental Psychology 22, 681-685 (1986)

14. Kruger, R., Carpendale, S., Scott, S.D., Greenberg, S.: Roles of Orientation in Tabletop Collaboration: Comprehension, Coordination and Communication. In: Proc. of CSCW, pp. 501-537. ACM (2004)

15. Marshall, P., Fleck, R., Harris, A., Rick, J., Hornecker, E., Rogers, Y., Yuill, N., Dalton, N.S.: Fighting for Control: Children's Embodied Interactions When Using Physical and Digital Representations. In: CHI, pp. 2149-2152. ACM (2009)

16. Marshall, P., Morris, R., Rogers, Y., Kreitmayer, S., Davies, M.: Rethinking 'Multi-User': An In-the-Wild Study of how Groups Approach a Walk-Up-and-Use Tabletop Interface. In: Proc. of CHI, pp. 3033-3042. ACM (2011)

17. Marshall, P., Rogers, Y., Pantidi, N.: Using F-formations to Analyse Spatial Patterns of Interaction in Physical Environments. In: Proc. of CSCW, pp. 445-454. ACM (2011)

18. Nacenta, M.A., Pinelle, D., Stuckel, D., Gutwin, C.: The effects of interaction technique on coordination in tabletop groupware. In: Proc. of GI, pp. 191-198. ACM (2007)

19. Olson, I.C., Leong, Z.A., Wilensky, U., Horn, M.S.: "It's just a toolbar!" Using Tangibles to Help Children Manage Conflict Around a Multi-Touch Tabletop. In: Proc. of TEI, pp. 29-36. ACM (2011)

20. Pawar, U.S., Pal, J., Gupta, R., Toyama, K.: Multiple mice for retention tasks in disadvantaged schools. In: Proc. of CHI, pp. 1581-1590. ACM (2007)

21. Piper, A.M., O’Brien, E., Morris, M.R., Winograd, T.: SIDES: A cooperative tabletop computer game for social skills development. In: Proc. of CSCW, pp. 1-10. ACM (2006)

22. Rick, J., Harris, A., Marshall, P., Fleck, R., Yuill, N., Rogers, Y.: Children designing together on a multi-touch tabletop: an analysis of spatial orientation and user interactions. In: Proc. of IDC, pp. 106-114. ACM (2009)

23. Rogers, Y., Lim, Y.-K., Hazlewood, W., Marshall, P.: Equal Opportunities: Do Shareable Interfaces Promote More Group Participation Than Single User Displays? HumanComputer Interaction 24, 79-116 (2009)

24. Scott, S., Sheelagh, M., Carpendale, T., Inkpen, K.: Territoriality in collaborative tabletop workspaces. In: Proc. of CSCW, pp. 294-303. ACM Press (2004)

25. Segal, L.: Designing Team Workstations: The Choreography of Teamwork. In: Local Applications of the Ecological Approach to Human-Machine Systems (1995)

26. Suzuki, H., Kato, H.: Interaction-level support for collaborative learning: AlgoBlock-an open programming language. In: Proc. of CSCL, pp. 349-355 (1995)

27. Tang, A., Tory, M., Po, B., Neumann, P., Carpendale, S.: Collaborative coupling over tabletop displays. In: Proc. of CHI, pp. 1181-1190. ACM (2006)

28. Teasley, S.: The Role of Talk in Children's Peer Collaborations. Developmental Psychology 1, 207-220 (1995)

29. Yamashita, N., Kuzuoka, H., Hirata, K., Aoyagi, S., Shirai, Y.: Supporting fluid tabletop collaboration across distances. In: Proc. of CHI, pp. 2827-2836. ACM (2011) 